\title{
Young Children Associate Novel Words With Complex Objects Rather Than Salient Parts
}

\author{
George Hollich \\ Purdue University
}

\author{
Roberta M. Golinkoff \\ University of Delaware
}

\author{
Kathy Hirsh-Pasek \\ Temple University
}

\begin{abstract}
How do children learn associations between novel words and complex perceptual displays? Using a visual preference procedure, the authors tested 12- and 19-month-olds to see whether the infants would associate a novel word with a complex 2-part object or with either of that object's parts, both of which were potentially objects in their own right and 1 of which was highly salient to infants. At both ages, children's visual fixation times during test were greater to the entire complex object than to the salient part (Experiment 1) or to the less salient part (Experiment 2) - when the original label was requested. Looking times to the objects were equal if a new label was requested or if neutral audio was used during training (Experiment 3). Thus, from 12 months of age, infants associate words with whole objects, even those that could potentially be construed as 2 separate objects and even if 1 of the parts is salient.
\end{abstract}

Keywords: word learning, constraints, whole object bias
As Quine (1960) observed, the task of learning a word presents an infinite array of possible word-referent links. A word such as bottle could refer to the nipple, to the plastic base, or to the whole bottle including both of these parts. It also could refer to sucking or even the process of feeding (see Bloom, 2000). Nonetheless, despite many possible misinterpretations, children generally sort out the correct meanings. How?

One seemingly obvious solution to this problem is that children will make an educated guess. Indeed, one branch of research in developmental psychology has sought to identify the heuristics that children use to limit their hypotheses about the meaning of a new word (e.g., Clark, 1983; Golinkoff, Mervis, \& Hirsh-Pasek, 1994; Markman \& Hutchinson, 1984; Nelson, 1988; Waxman \& Kosowski, 1990). One such tendency that Macnamara (1972, 1982, Markman (1989), and others (Golinkoff et al., 1994) have noted is that children appear to guess that labels refer to whole

George Hollich, Department of Psychological Sciences, Purdue University; Roberta M. Golinkoff, School of Education, University of Delaware; Kathy Hirsh-Pasek, Department of Psychology, Temple University.

This research was supported by a National Science Foundation Grant SBR9601306 to Roberta M. Golinkoff and Kathy Hirsh-Pasek and a National Institute of Child Health and Human Development grant to Kathy Hirsh-Pasek. We gratefully acknowledge the help of all the parents and infants who participated in this research. We are also fortunate to have had many wonderful undergraduate and graduate students assist us in our labors and enrich our work. Special thanks goes to Karen Arnold, Camille Rocroi, and Elizabeth Hennon for their roles in running participants and helping with earlier versions of this article.

Correspondence concerning this article should be addressed to George Hollich, Department of Psychological Sciences, Purdue University, 703 Third Street, West Lafayette, IN 47907-2004. E-mail: ghollich@ purdue.edu objects rather than actions, attributes, or parts of objects. Markman (1989) called this heuristic the whole object bias. For example, in a seminal study, Woodward (1993) presented 18-month-old children with a novel word and two possible referents. One referent was a visually attractive display representing an event (e.g., brightly colored dye diffusing through water); the other was a novel object in a static display. Despite a salience preference for the event, the children looked at the object more when they heard a novel noun.

There are a few problems with this whole object bias as a solution to the word-learning dilemma. First, most of the evidence for this tendency is taken from children 18 months of age and older-most typically age 2 years (Kersten \& Smith, 2002; Markman \& Wachtel, 1988; Saylor, Sabbagh, \& Baldwin, 2002). Very little is known about how younger children interpret new labels, if their "educated" guesses would be less well informed, or if those guesses would hold under the same types of situations.

Second, previous work has used a highly restricted set of stimuli that potentially favors a "whole" interpretation, especially with regard to parts. That is, the stimuli used previously were objects for which parts were not particularly salient, and those parts were not necessarily distinct from the object, as would be the case for parts that separate from an object itself. Yet children do learn words for complex objects made up of separable parts (e.g., flowers with petals, pens with caps, shoes with laces). For such objects, including a myriad of toys, those parts may draw attention (e.g., the wiggly antenna on a caterpillar toy), they may have their own labels (e.g., leaves on a tree), and they may separate from the whole at times and be easily construed as objects in their own right (e.g., the ear on Mr. Potato Head). Nothing is known about how children interpret labels in the presence of such complex novel objects or even if anything like a whole object bias would obtain in such a case. 
Finally, the biggest problem with using the whole object bias as a mechanism for explanation is that it is more a description than an explanation. That children, ages 2 years and above, apparently prefer to label wholes under a restricted set of circumstances does not actually explain how or why such a bias arises in the first place. Although the whole object bias could result from the workings of a hardwired linguistic constraint (as is often portrayed), this apparent bias could also result from the workings of well-established general mechanisms of memory and attention such as orientation to novelty, blocking (when one association "blocks" the learning of additional associations), or overshadowing (when one association is "overshadowed" by another, more salient, association). The tendency to label whole objects could also be learned, as has been suggested for other biases such as the tendency to extend words on the basis of shape (Merriman, 1999; Smith, 1999). Likewise, the whole object bias could be the result of the workings of other domain-specific skills, such as sensitivity to social intent and categorization ability, or simply a perceptual bias toward wholes. Given these many possible explanations for the tendency of older children to label whole objects, we suggest that it is almost certainly premature to definitively decide which of these mechanisms (or what combination of them) is responsible.

Bearing in mind that researchers have used a limited range of ages, stimuli, and methods to test children's tendency to associate words with whole objects, in this work we adopted an exploratory approach and sought to establish when younger children are exhibiting this tendency and under what situations and stimuli it seems to hold (as suggested by Deák, 2000). Specifically, in the current experiments, we asked what happens when 12- and 19month-olds are given a label for a complex multipart object that has a salient, separable part. In doing so, we tested the idea that the tendency to label wholes could result from the salience of contiguous objects. Nineteen-month-olds were selected because this is close to the youngest age at which it is known that some form of the tendency to label wholes exists (Woodward, 1993). If these children fail to associate a novel word with the complex object, it would limit the extendibility of previous findings of a tendency to label wholes at this age. Twelve-month-olds were selected because they are among the youngest infants that have been shown to learn words in a laboratory setting (Hollich, Hirsh-Pasek, \& Golinkoff, 2000; Pruden, Hirsh-Pasek, Golinkoff, \& Hennon, 2006), and it is worthwhile to know if even younger children approach the wordlearning task with this tendency.

\section{Prior Work}

A majority of words in the vocabularies of infants are at the basic level of categorization: nouns such as bike or bottle, highlighting individual entities and their global shapes (Smith, 1999) rather than their constituent parts, attributes, or accompanying actions (Fenson et al., 1994; Mervis, 1990). A child who guesses that novel words highlight whole objects over parts, attributes, or actions would be at a considerable advantage in the task of acquiring a noun lexicon. There is a large body of evidence for the existence of just such a tendency in children 18 months of age and older (Kersten \& Smith, 2002; Landau, Smith, \& Jones, 1988, 1992; Markman \& Wachtel, 1988; Saylor et al., 2002; Soja, Carey, \& Spelke, 1991; Woodward, 1993).
Some of the evidence focuses on children's tendency to associate a label with a whole object rather than that object's attributes (Baldwin, 1989; Smith, Jones, \& Landau, 1992). Thus, Markman and Wachtel (1988) tried to teach an adjective to 3-year-olds by presenting them with a novel object and telling them, "This is chrome." Children chose a similarly shaped object made of a different material as a referent for the word. They did so despite the absence of an article ("a" or "the") before the new term, which should have tipped them off that the novel word was not a count noun. This bias toward wholes over attributes was found at younger ages by Hall, Waxman, and Hurwitz (1993), who showed that 2-year-olds interpret a novel word as a label for an object, even in the presence of unambiguous cues that the word was an adjective, mass, or property term (e.g., "give me the blickish one"). Likewise, Imai and Gentner (1997) found that, despite being reared in a language community that classifies many more items as substances, even Japanese 2-year-olds interpreted syntactically neutral labels as names for complex objects that had parts and functions.

Children 18 months of age and older also seem predisposed to learn the name for a whole object before the names for any parts. For example, Markman and Wachtel (1988) found that their participants could only learn a part name if those participants already knew the name for the whole object. Likewise, Saylor et al. (2002; Saylor \& Sabbagh, 2004; see also Masur, 1997) found that explicit contrast, either linguistic or gestural, was essential to learning a part name for 3- and 4-year-olds-even when the children knew the name of the object. Thus, the experimenter had to label the whole object first (e.g., "See the fish? What color is the dorsal?") or gesture to the entire fish first (e.g., "See this [circular motion]? What color is the dorsal [point to part]?"). Similarly, Kobayashi (1998) found that while $2 \frac{1}{2}$-year old children could learn a novel word for an object part, they did so only if the experimenter acted on the part during training.

Such results need to be tempered by the fact that in these studies, it was likely easiest to associate the word with the whole object. Actions are transient, attributes are not especially salient, and parts are often not perceptually distinct enough to draw a clear association. Gentner $(1978,1982,2006)$ has described such a position in her natural partitions hypothesis. She noted that because nouns commonly refer to whole objects, and objects are naturally perceived as units, nouns are relatively easy to link to labels. The work of Huttenlocher and Smiley (1987) also supports the notion that nouns for objects are simply easier for children to learn. These authors reported that young children produced nouns in an adultlike way from the beginning of word learning, using them in an appropriate and wide range of contexts.

It is possible, then, that the apparent tendency to label whole objects may be a result of the fact that wholes are perceptually more salient-processed as distinct units in a way that parts are not. A long tradition of research in infant perception elucidates the factors that lead infants to perceive object unity: common fate, similar texture, contiguous boundaries, and so forth (Carey \& Xu, 2001; Spelke, 1990). Much of this work suggests that infants are biased to focus on discrete contiguous objects. This has been demonstrated by Shipley and Shepperson (1990), who found that even when explicitly asked to count only wholes (e.g., "count the forks"), 3-year-olds tended to include separate parts (of a broken fork) in their count as well. Likewise, in support of this idea, Giralt 
and Bloom (2000) found that while 3-year-olds had no trouble counting objects, they had much more difficulty counting the "handles" on three novel objects.

Such work suggests that if the "wholes" were not contiguous, if the parts could be made more salient, infants might not exhibit a tendency to associate labels with wholes at all. To test this idea, in the current experiments, the experimenter labeled a novel two-part, separable object while taking it apart and putting it back together. In this manner, we attempted to bias infants toward a "part" interpretation by offering the label more when the object was apart than when it was together. This created a situation in which the perceptual cues directly contradicted a whole object interpretation and, instead, highlighted a patterned and separate part, designed to be salient to the child, that could plausibly be interpreted as an object in its own right. We expected that because infants are so taken by object salience (Hollich et al., 2000; Pruden, Hirsh-Pasek, Golinkoff, \& Hennon, 2006), they would fail to learn a label for the whole object and instead associate the label with the perceptually interesting and separate part. If so, this would suggest that the tendency to associate words with whole objects may partially result from the perceptual salience of contiguous objects.

\section{Experiment 1}

\section{Method}

Because preverbal children cannot be asked to describe what they think a word refers to, the interactive intermodal preferential looking paradigm was used (Hollich, Hirsh-Pasek, \& Golinkoff, 1998; Hollich et al., 2000). This experimental paradigm is based on the intermodal preferential looking paradigm (Golinkoff, HirshPasek, Cauley, \& Gordon, 1987; Hirsh-Pasek \& Golinkoff, 1996, Baldwin's (1991) bucket task, and Fagan's (Fagan, Singer, Montic, \& Shepard, 1986) infant intelligence test. It has proven highly reliable in testing young children's comprehension of both familiar and novel words (Hollich et al., 1998). In the procedure, pairs of stimuli are affixed to a rotating board, and the experimenter asks participants to look at or find a particular object on the side of the board facing toward them (see Figure 1). Because the procedure uses a response that is already in children's behavioral repertoirevisual fixation-as its dependent variable, it can be used to test children at earlier ages than can procedures that require a verbal or acted-out response.

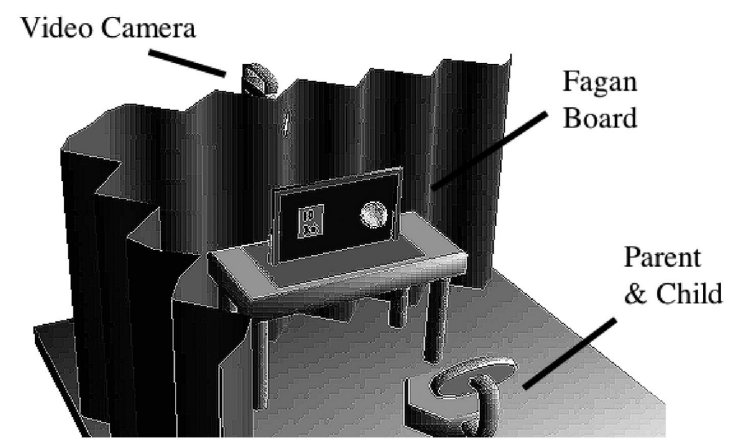

Figure 1. The interactive intermodal preferential looking procedure.
Participants. A total of 48 infants, balanced for gender, participated. The 12-month-olds $(n=28)$ had a mean age of 12.55 (range: $11.85-13.23)$. The 19-month-olds $(n=20)$ had mean age of 18.97 (range: 18.16-20.13). Participants were recruited from local birth announcements or purchased mailing lists and were tested at two university laboratories. This resulted in a sample for this experiment and all subsequent experiments that was predominately White and middle class, although detailed race/ethnicity and parental education and occupation data were not collected. As measured by the MacArthur Communicative Development Inventory (Fenson et al., 1994), the mean numbers of words produced were $10.16(S E=2.60)$ and $70.00(S E=20.14)$ for the 12 - and 19-month-olds, respectively. Although the total comprehension of the 19-month-olds was too high to measure, the comprehension of the 12-month-olds was 92.68 ( $S E=13.33$ ) words. The caregivers of all infants signed an approved human participants consent form.

Stimuli and apparatus. We created four sets of multipart stimuli, two familiar sets and two novel sets. The familiar sets consisted of common two-part objects with distinct (possibly salient) parts (phone with handset, shoe with laces, cup with lid, and bottle with nipple). Novel stimuli were $1 / 2$-in. thick wood cutouts (see Figure 2) that could separate into two pieces. The pieces fit together like a simple puzzle (aided by Velcro) and were light enough that a 12-month-old could pull them apart easily. The base of the objects was painted a single solid color (red or green), and the part had markings in addition to the solid color. The red object had light blue polka dots; the green object had yellow stripes. These markings represented an attempt to heighten the salience of the parts and increase the likelihood that infants would associate the word with them. Prior work has demonstrated that in the absence of cues to the contrary, infants will associate words with the most salient object (Hollich et al., 2000).

The testing apparatus consisted of a rotating black board $(40 \times$ $50 \mathrm{~cm}$ ) resting on a table positioned between the experimenter and the infant participant (see Figure 1). Stimuli could be affixed to the board by strips of Velcro, $30 \mathrm{~cm}$ apart. The board was positioned $75 \mathrm{~cm}$ away from the infant so that the stimuli on the board were out of the infant's reach. The board could be rotated almost $180^{\circ}$, allowing the stimuli to be brought completely into and out of the infant's view for timed intervals, and was high enough for the experimenter to hide behind it completely during test trials. A video camera on a tripod above and behind the experimenter recorded the participant's attention to the board as well as the position of the stimuli, which could be seen reflected in a mirror on the wall behind the participant.

Procedure. Testing began when the infant was seated comfortably on a caregiver's lap facing the testing apparatus and experimenter. The caregivers were either blindfolded or instructed to close their eyes during all test trials. The sequence of trials is depicted in Table 1. Children first saw a pair of familiar objects (phone and shoe, cup and bottle) during the exploration phase. We ran these familiar trials to familiarize infants with the preferential looking task and to introduce objects that can be seen both apart and together. During a 26-s exploration phase, children were allowed to handle the objects one at a time, both apart and together (e.g., the phone was shown on and off its cradle, the cup was handled with and without the lid). Then, during the test trials, the two objects were affixed to the board; the experimenter turned the board so that it faced the child, ducked behind it, and asked for one 

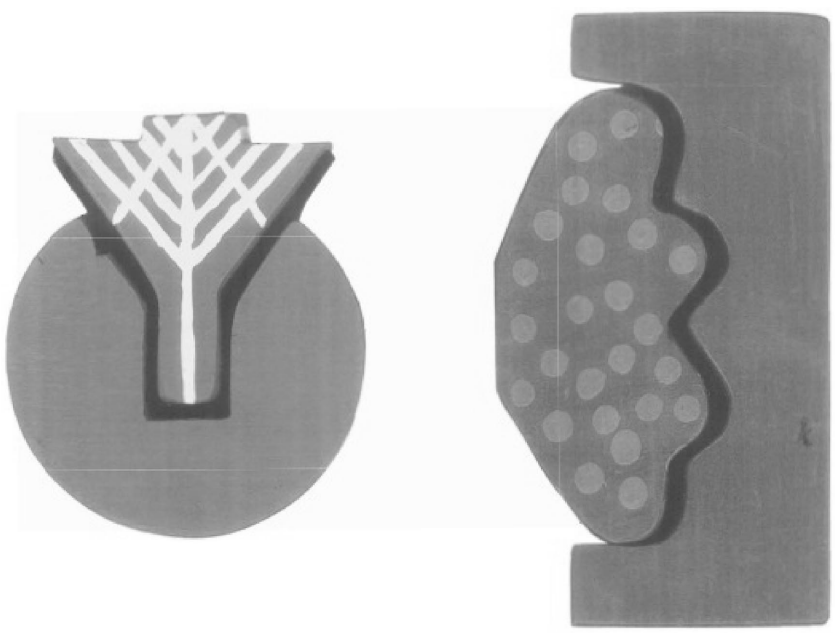

Figure 2. The two novel objects used in the experiments.

of the familiar objects three times during a 6-s trial, using a randomized assortment of token-final carrier phrases (e.g., "Where's the shoe? Show me the shoe! Find the shoe!"). The experimenter coordinated the presentation of these phrases using a digital stopwatch.

Next, the novel stimuli were introduced. During the 26-s exploration phase, the child was encouraged by the experimenter to move the pieces apart and together. A 6-s salience trial with the whole object and its attractive part affixed to the board then determined each child's baseline preference (e.g., "Look up here! What do you see?"). During training, the experimenter labeled the novel object with a nonsense word (either modi [modaj] or dawnoo [danu]) seven times, using token-final sentence frames (e.g., "Look at the modi! See the modi?"; "It's a modi!"), while pulling the pieces apart and reattaching them. In this manner, the label did not consistently co-occur with the object's pieces being together. This manipulation was done for the same reason that one part was patterned: to bias children toward a part interpretation of the novel word. In fact, the label was rarely uttered while the object was together (less than $15 \%$ of the time, according to post hoc coding of a subset of the data), minimizing the chance of a whole object interpretation. This coding indicated that the majority of labels were produced when the objects were coming apart $(45 \%)$ or coming together $(24 \%)$. In addition, the experimenter maintained visual fixation on the infant's eyes to eliminate the possibility that the infant would use the experimenter's gaze to determine the intended referent of the label.

The testing phase had four, 6-s trials. During the first two test trials, the experimenter hid behind the testing board on which the infant saw the patterned part and the whole object side by side (see Figure 3). The experimenter requested that the child look at the target object, "[Child's name], where's the modi? Can you find the modi?" If children affixed the label to the whole object, we hypothesized that they should look at the whole object more than at the part. If, however, children affixed the name to the part, they should either look equally to both objects (because both contained the part) or look significantly more at the part by itself. The tendency to look longer at a labeled object has been well established (Hollich et al., 1998; Schafer \& Plunkett, 1998).
In the next two trials, we conducted a control to assess whether word learning had really occurred. In the new-label trial, a new label (glorp or blicket) was offered. Thus, for example, infants were asked to look at the "glorp" rather than at the "modi." Consistent with previous work (Hollich et al., 2000), if infants had specifically connected the label to the whole object, we expected they should look less at it on these trials when an alternative label was requested. ${ }^{1}$ The final recovery trial ruled out that children had just looked away from the whole object out of fatigue by asking the child to again look at the whole object-the modi. Thus, a pattern of decreased looking on the new-label trials and increased looking on the recovery trials would insure that the infant's mapping was specific to the label and object used and provides a more stringent test of the word-learning hypothesis.

\section{Results and Discussion}

Observers, who were blind to the condition being run, coded visual fixations offline from the videotape. Interobserver reliability was obtained by means of random recodings of $10 \%$ of the children. In all cases, the correlation between coders was above .98 .

Familiar trials. The mean looking times on the familiar trials for the 19-month-olds were $3.58 \mathrm{~s}(S E=0.18)$ and $1.92 \mathrm{~s}(S E=$ $0.18)$ to the target and nontarget, respectively. The looking times for the 12 -month-olds were $3.09 \mathrm{~s}(S E=0.15)$ and $1.96 \mathrm{~s}(S E=$ 0.13 ) to the target and nontarget, respectively. $T$ tests revealed that both the 19-month-olds, $t(19)=4.85, p<.0001$, and the 12 month-olds, $t(27)=4.47, p<.0001$, looked significantly longer at the requested object when familiar objects were used. Furthermore, not only were the effect sizes large (Cohen's $d \mathrm{~s}=1.0$ and 0.8 , respectively), this finding was also not confined to a few "expert" responders: for 12-month-olds 23 out of 28 (sign test: $p<$ .001 ) and for 19-month-olds 17 out of 20 (sign test: $p<.003$ ) looked longer at the requested object. In contrast, an unpaired $t$ test conducted on the difference scores (target minus nontarget) found no evidence of a significant shift in looking preferences across ages, $t(46)=1.27, p=.21, d=0.2$. Thus, it appears that infants at both ages could correctly locate the requested familiar objects, and there were not large differences in performance between ages on the familiar trials.

Novel trials. These trials were designed to be a test of the possible perceptual nature of the tendency to label whole objects. Would infants assume a whole object interpretation of a new label even when shown a multipart, perceptually separable object? They did. The mean looking times to the whole, to the part, and their differences (whole minus part) are presented in Table 2 for each of the novel trials and across the two age groups. Also presented in Table 2 is the result of a matched-pairs $t$ test comparing looking to the whole versus part. Table 2 indicates that both the 12- and 19-month-olds looked significantly longer at the whole when the label was requested (in the test and recovery trials) but not in the salience or new-label trials.

\footnotetext{
${ }^{1}$ Although the logic of this is similar to Markman and Wachtel's (1988) principle of mutual exclusivity, the infant does not have to associate the new label with the part or with another object. It is enough that the original looking preference decreases.
} 
Table 1

Experiment Design: Sequence and Description of Trial Types

\begin{tabular}{|c|c|c|}
\hline Trial type & Duration & Description \\
\hline \multicolumn{3}{|r|}{ Familiar object trials } \\
\hline Exploration $(\times 2)$ & $26 \mathrm{~s}$ each & Infant haptically explores each of two familiar objects (e.g., phone and shoe) separately \\
\hline Test $(\times 2)$ & $6 \mathrm{~s}$ each & Both stimuli placed on the board; specific request made (e.g., "Can you find the shoe?") \\
\hline \multicolumn{3}{|r|}{ Novel object trials } \\
\hline Exploration $(\times 1)$ & $26 \mathrm{~s}$ & Infant haptically explores the novel object and sees that it is separable \\
\hline Salience & $6 \mathrm{~s}$ & Whole novel object and one of its parts on the board; neutral request made (e.g., "What do you see?") \\
\hline Training & $20-30 \mathrm{~s}$ & $\begin{array}{l}\text { Experimenter assembles and disassembles the novel object and labels it seven times, (e.g., "The modi! I've got the } \\
\text { modi!), maintaining eye contact with the infant }\end{array}$ \\
\hline Test $(\times 2)$ & $6 \mathrm{~s}$ each & $\begin{array}{l}\text { Whole novel object and one of its parts on the board; specific request for labeled object (e.g., "Where's the } \\
\text { modi?") }\end{array}$ \\
\hline New label & $6 \mathrm{~s}$ & Whole object and one of its parts on the board; request for object using new word (e.g. "Find the glorp") \\
\hline Recovery & $6 \mathrm{~s}$ & Whole object and one of its parts on the board; request for originally labeled object (e.g., "Look at the modi") \\
\hline
\end{tabular}

Note. Each infant was exposed to two familiar object blocks and two novel object blocks for a total of four trial blocks, each using different sets of objects: phone/shoe, lidded cup/bottle, and two multipart novel objects. The order was always familiar/novel/familiar/novel. However, the specific stimuli and the side of target objects were counterbalanced across infants.

It might seem surprising that infants showed no salience preference toward the whole object during the salience trial, but recall that both sides displayed the patterned part. Subsequent testing (see Experiment 2) indicated that infants did find the patterned part very interesting. The fact that children did not have a preference for the patterned part or the whole object during the salience trial reinforces the importance of the label in our findings. The preference for the whole object after label training could not be the result of an a priori perceptual preference for the whole object. Thus, words, per se, did not simply increase attention to the whole object. Otherwise, infants should have looked more at the whole object on the new-label trials as well.

To demonstrate the specific effect of requesting the label, we conducted an additional analysis that looked for changes in infants' looking preferences across trials. For the purpose of this analysis, the looking times to the part were subtracted from the looking times to the whole to produce a mean difference in looking times. A positive number in this difference score indicated how much more infants were looking at the whole than the part on any

\section{Experiment 1: Part Condition}
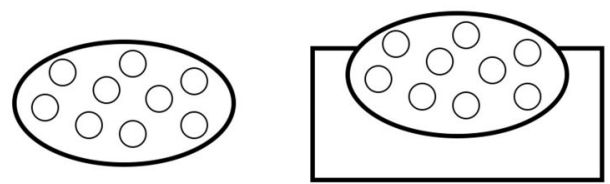

Experiment 2: Base Condition

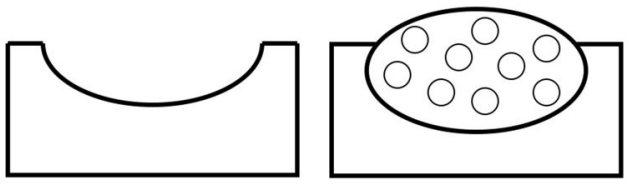

Figure 3. Sample displays from the experiments. given trial. This subtraction was done because we wanted to look for changes in looking preference rather than changes in raw looking. Raw looking to one object is meaningless without a comparison with the visual fixation time to the other object. These difference scores were submitted to a repeated measures analysis of variance (ANOVA) with age as a between-subjects factor and trial type (salience/test/new label/recovery) as the repeated factor. ${ }^{2}$ Neither the main effect of age, $F(1,46)=0.01, p=.908$, nor the Age $\times$ Trial interaction, $F(3,138)=0.11, p=.953$, were significant. However, the trial factor was highly significant, $F(3$, $138)=6.33, p=.0005$. Subsequent Fisher post hoc tests (with a critical difference at the $p<.05$ level of 0.477 ) revealed that the significant trial factor was a result of the test trials differing significantly from the salience trials $(p=.004)$ and the new-label trials $(p=.0005)$ and the recovery trials differing from the salience trials $(p=.015)$ and the new-label trials $(p=.002)$.

In addition, to ensure that the data from the 19-month-olds were not responsible for these significant effects, we conducted a separate ANOVA solely on the data from the 12-month-olds, with equally significant results. In addition to these ANOVAs, an analysis of individual performances revealed that for 12-montholds 19 of the 28 (sign test: $p \leq .243$ ) and for 19-month-olds 17 of the 20 (sign test: $p \leq .003$ ) looked more at the whole object during test trials than during salience trials. The results of these ANOVAs and the sign tests suggest that infants (even 12-month-olds) looked at the whole object significantly more when a label was used than when a label was not. Similarly, infants looked longer at the whole object during all test trials, including the recovery trials, than they did on the new-label or salience trials.

This pattern of results cannot be easily explained by habituation. Children looked evenly between the whole and the part on the salience trials, then looked more at the whole object when it was

\footnotetext{
${ }^{2}$ Prior to this analysis, we ran a $t$ test to determine whether any significant differences existed between the participants from either lab. No significant differences were found.
} 
Table 2

Experiment 1: Mean Looking Times (in Seconds, With Standard Errors in Parentheses) to Wholes and Patterned Parts Across Trials and Age Groups

\begin{tabular}{lcccc}
\hline \multicolumn{1}{c}{ Trial } & Whole & Patterned part & Difference & $\begin{array}{c}\text { Effect size } \\
(d)\end{array}$ \\
\hline \multicolumn{5}{c}{ 12-month-olds $(n=28)$} \\
Salience & $2.61(0.12)$ & $2.32(0.11)$ & 0.29 & 0.5 \\
Test & $2.73(0.12)$ & $1.79(0.07)$ & $0.94^{* * * *}$ & 1.9 \\
New label & $1.88(0.17)$ & $1.68(0.17)$ & 0.20 & 0.2 \\
Recovery & $2.43(0.22)$ & $1.56(0.15)$ & $0.87^{* * * *}$ & 0.9 \\
\hline
\end{tabular}

19-month-olds $(n=20)$

\begin{tabular}{lllll} 
Salience & $2.81(0.13)$ & $2.55(0.14)$ & 0.26 & 0.4 \\
Test & $2.90(0.15)$ & $1.84(0.09)$ & $1.06^{\text {**** }}$ & 2.1 \\
New label & $2.02(0.23)$ & $1.99(0.20)$ & 0.03 & 0.0 \\
Recovery & $2.04(0.27)$ & $1.16(0.20)$ & $0.88^{* *}$ & 0.8 \\
\hline
\end{tabular}

Note. Significance levels are according to $t$ test against chance responding. Effect sizes were calculated as follows: $d=$ difference/(average $S D$ ). ${ }^{*} p<.05 .^{* * * *} p<.005$.

requested by name during the new-label trials, then looked evenly again on the new-label trials, and then looked longer at the whole object on the recovery trials. This distinctive pattern of even/ biased/even/biased looking in infants of two different ages across identical time periods would not be predicted by any theory of habituation of which we are aware. In addition, this pattern of results (greater looking at the whole object when the label is used) also rules out the possibility that neither the whole nor the part was taken as the reference for a novel label. That is, one possibility is that the ambiguity of the stimuli (one object or two?) combined with the ambiguity of the training (apart and together) might have lead the infants to connect the label with neither the part nor the whole. However, had that been the case, looking preferences should have remained the same throughout the trials or steadily decreased. This did not happen. We also note that this pattern of results demonstrates that the infants did not simply prefer the "bigger" of the two displays: It was only when a label was offered that the preference for the whole was seen.

\section{Experiment 2}

In Experiment 1, despite perceptual cues to the separate nature of the object stimuli, infants' looking responses suggested that they associated the label with the whole object over the patterned part. Nonetheless, the possibility remains that participants had associated the label not to the whole but to the base part, as if it were a container and the multicolored piece simply its contents. Although we made every effort to make the patterned part more salient than the base, the results from Experiment 1 are compatible with the interpretation that it was the base that had been associated with the novel word, not the whole object per se. Looking times during test trials were longer toward the stimulus in which the base was present (the whole object) than the one in which it was not (the part).

To rule out this possibility, we conducted a second experiment with the whole object versus the base part during testing. Exper- iment 2 was designed to see whether the results of Experiment 1 were attributable to a preference for associating the novel label with the base of the novel object or with the whole object. If the results from Experiment 1 were attributable to the association between the novel word and the base, then the prediction would be that looking times should be equal to the base and the whole object during test trials (or even favor the base). However, if the results of Experiment 1 were attributable to the action of a systematic bias toward labeling the whole object, then during test trials, infants should still show even more of a preference for the whole object. As an added benefit, this experiment also provided a means to test whether children found the patterned part truly salient. That is, in Experiment 1, the patterned part was present on both sides of the display. It was thus impossible to directly measure any prior preference for the patterned part. In Experiment 2, the patterned part was not present on both sides of the display, allowing us to check for a salience preference. The indication of a preference would mean that the analysis must correct for this preference as well.

\section{Method}

Participants. A total of 48 different infants, balanced for gender, participated. The 12-month-olds $(n=28)$ had a mean age of 12.43 (range: $11.85-13.16)$. The 19-month-olds $(n=20)$ had a mean age of 19.03 (range: 18.10-20.30). As tested using the MacArthur Communicative Development Inventory (Fenson et al., 1994), the mean numbers of words produced were 5.70 ( $S E=$ $1.50)$ and $98.81(S E=26.08)$ for the 12- and 19-month-olds, respectively. The comprehension of the 12-month-olds was 65.23 $(S E=12.56)$ words.

Stimuli and apparatus. The stimuli and apparatus were identical to those in Experiment 1.

Procedure. All phases of the protocol proceeded identically to that in Experiment 1 except that during test trials, the stimuli presented were the whole object and the base part (see Figure 3).

\section{Results and Discussion}

Familiar trials. The mean looking times on the familiar trials for the 19-month-olds were $3.38(S E=0.16)$ and $2.20(S E=0.14)$ to the target and nontarget, respectively. The looking times for the 12-month-olds were $2.63(S E=0.15)$ and $2.33(S E=0.13)$ to the target and nontarget, respectively. $T$ tests against chance revealed that the 19-month-olds, $t(19)=4.09, p=.0006$, but not the 12-month-olds, $t(27)=1.30, p=.205$, looked longer at the requested object on the familiar trials. Furthermore, raw numbers revealed that for 12 -month-olds 18 out of 28 ( $\operatorname{sign}$ test: $p \leq .19$ ) and for 19-month-olds 18 out of 20 (sign test: $p<.001$ ) looked longer at the requested object. An unpaired $t$ test conducted on the difference scores (target minus nontarget) indicated that there was a significant difference in looking preferences between the ages, $t(46)=2.37, p=.022$. Thus, it appears that the 12-month-olds in this experiment were less adroit during the familiar trials than were the 19-month-olds. Nonetheless, as is shown below, their performance on the novel trials closely mirrored that of the 19-montholds.

Novel trials. These trials were conducted to see if infants would attach the label to the base part rather than the whole 
complex object. They did not. The mean looking times to the whole and to the part and their differences are presented in Table 3 for each of the novel trials and across the two age groups. Also presented in Table 3 is an analysis against chance responding. Table 3 indicates that both the 12- and 19-month-olds again looked longer at the whole specifically when the label was requested (in the test and recovery trials) but not in the new-label trials, although they also looked significantly more at the whole during salience trials. Given that the plain red base was paired with a whole object that included the interesting part, this result is most likely attributable to the inherent interest of the part.

Again, to further demonstrate the specific effect of requesting the label, we conducted an additional analysis comparing infants' looking preferences across trials. As before, the looking times to the part were subtracted from the looking times to the whole to produce a mean difference in looking times. A $2 \times 4$ (Age $\times$ Trial: salience/test/new-label/recovery) mixed ANOVA was conducted on these difference scores. The age factor revealed no significant effects, $F(1,46)=0.05, p=.817$, whereas the trial factor was significant, $F(3,138)=14.70, p<.0001$. No Age $\times$ Trial interaction was observed, $F(3,138)=0.418, p=.740$. Subsequent Fisher post hoc tests (with a critical difference at the $p<.05$ level of 0.555) revealed that the significant trial factor was a result of the test trials differing significantly from the new-label trials $(p<.0001)$ and the recovery trials differing from the new-label trials $(p<.0001)$. Thus, infants looked at the whole object significantly more when the label was requested than they did when it was not. ${ }^{3}$ However, in contrast to the previous experiment, the salience trials also differed significantly from the new-label trials $(p<.0001)$. The raw numbers also reflected this salience preference: for 12 -month-olds only 15 of the 28 ( sign test: $p \leq .85$ ) and for 19-month-olds 16 of the 20 (sign test: $p \leq .01$ ) looked more at the whole object during test trials than during salience trials. This is likely because the base was intentionally created to be inherently boring and because the patterned part was "part" of the whole object.

Table 3

Experiment 2: Mean Looking Times (in Seconds, With Standard Errors in Parentheses) to Wholes and Base Parts Across Trials and Age Groups

\begin{tabular}{lcccc}
\hline \multicolumn{1}{c}{ Trial } & Whole & Base part & Difference & $\begin{array}{c}\text { Effect size } \\
(d)\end{array}$ \\
\hline \multicolumn{4}{c}{ 12-month-olds $(n=28)$} \\
Salience & $3.24(0.13)$ & $2.02(0.13)$ & $1.22^{* * * * *}$ & 1.7 \\
Test & $3.10(0.13)$ & $1.57(0.12)$ & $1.53^{* * * * *}$ & 2.3 \\
New label & $1.97(0.16)$ & $2.13(0.20)$ & -0.16 & 0.2 \\
Recovery & $2.21(0.14)$ & $1.24(0.15)$ & $0.97^{* * *}$ & 1.3 \\
\hline
\end{tabular}

19-month-olds $(n=20)$

\begin{tabular}{lllll} 
Salience & $3.32(0.13)$ & $2.31(0.12)$ & $1.01^{\text {*** }}$ & 1.5 \\
Test & $3.31(0.17)$ & $1.66(0.16)$ & $1.65^{\text {**** }}$ & 2.2 \\
New label & $2.19(0.26)$ & $2.42(0.30)$ & -0.23 & 0.2 \\
Recovery & $2.89(0.28)$ & $1.53(0.23)$ & $1.36^{\text {*** }}$ & 1.3 \\
\hline
\end{tabular}

Note. Significance levels are according to $t$ test against chance responding. Effect sizes were calculated as follows: $d=$ difference/(average $S D$ ). ${ }_{* * *} p<.006{ }^{* * * * *} p<.0001$.
Nonetheless, despite this salience preference, the performance on the test trials again indicates a specific link between label and whole object. This conclusion can be reached through an examination of the overall pattern of results. Although infants appeared to prefer to look at the whole object over the base part during the salience trials, training did have an effect. If the infants' attention on the test trials was driven purely by visual salience, then it should have made no difference what label the experimenter used; looking times should always have been overwhelmingly in favor of the more attractive, whole object stimulus. Although the results from both the salience and test trials could potentially be interpreted in this way, infants' behavior during new-label trials rules out this possibility. Infant looking times to the whole object decreased during the new-label trial and then returned to the original levels when the original label was used. The only explanation for both the lack of difference between test and recovery trials and the systematic difference between test, salience, and new-label trials is that infants' behavior in test trials reflected association of the word to the whole object. Thus, it appears that the bias to label the whole complex object was seen at the earlier age tested.

\section{Experiment 3}

Experiment 3 served as both a control for the use of language and a test of the genuine effects of labeling by presenting the same task without a linguistic label during training. If the presence of language - in the form of a label- biases children to interpret a novel label for a complex object as labeling the whole object, then in the absence of a label, no evidence of a bias should be found. Conversely, if language is not causing children to unify their perception of this complex object, but it is something about either the object itself during training or the social circumstances surrounding training that promotes attention to the whole, then we should find a bias toward the whole object over the parts, even in the absence of a label during training. Thus, although the previous experiments controlled for the presence of a specific label (i.e., through the use of the new-label and recovery trials), this experiment controlled for the act of labeling per se.

\section{Method}

Participants. The mean age of the 12-month-olds ( $n=26 ; 9$ boys and 17 girls) was 12.56 (range: 12.0-13.1). The 19-montholds ( $n=26 ; 13$ boys and 13 girls) had a mean age of 19.78 (range: 18.16-20.0). As tested using the MacArthur Communicative Development Inventory (Fenson et al., 1994), the mean number of words produced was $15.19(S E=3.85)$ and $103.31(S E=$ 22.56) for the 12- and 19-month-olds, respectively. The comprehension of the 12-month-olds was 110.27 ( $S E=13.15)$ words.

Stimuli and apparatus. The stimuli and apparatus were identical to those in Experiments 1 and 2.

Procedure. The procedure was the same as in the previous two experiments, with three exceptions: First, during the training phase, instead of labeling the object seven times, the experimenter

\footnotetext{
${ }^{3}$ Again, to ensure that the data from the 19-month-olds were not driving the effect, we conducted a separate analysis solely on the data from the 12 -month-olds, with equally significant results, $t(31)=4.45, p<.001$.
} 
said seven attention-getting sentences of approximately the same length and complexity (e.g., "Look at that!" instead of "Look at the modi!"). Second, during test trials, instead of the experimenter asking for the novel label (since none was introduced), the linguistic stimuli were sentences just as in the salience trials (e.g., "Look up here! What do you see? Oh, look!"). Third, because there were no test trials asking for a novel word, there were no new-label or recovery trials either.

\section{Results and Discussion}

The mean looking times to the whole object and the patterned part for each of the trials are shown in Table 4. A mixed (2 between $\times 2$ within: Age $\times$ Trial) ANOVA was conducted on the difference scores. The between factor again revealed no significant effect of age, $F(1,50)=0.61, n s$. For the within factor, there was no main effect of trial because the difference between the salience and test trials was also not significant, $F(1,78)=1.36, n s$. Thus, it appears that in the absence of a labeling situation, the preference for the whole object vanishes.

\section{Comparison Across Experiments 1 and 3}

We conducted a comparison of results in the two experiments in which the same object part was contrasted with the whole object to evaluate whether children in these experiments acted as differently as the results taken separately suggest. The analysis contrasted the change in looking between salience and novel trials in Experiment 1 (with labeling) versus Experiment 3 (without labeling). A threefactor (Age $\times$ Experiment $\times$ Change in Preference) ANOVA was run. The change in preference was calculated by subtracting the difference in looking times (target minus nontarget) in the salience trials from the difference in looking times during the new-label trials. The ANOVA revealed a significant main effect of experiment, $F(1,86)=3.94, p<.04$, with no effect of age, $F(1,86)=$ $0.001, p<.97$, or Age $\times$ Experiment interaction, $F(1,86)=0.32$, $p<.56$. Infants thus looked significantly longer at the whole object only when a label was provided during training. In Experiment 1 then, it was not the simple presence of actions and

Table 4

Experiment 3: Mean Looking Times (in Seconds, With Standard Errors in Parentheses) to Wholes and Patterned Parts Across Trials and Age Groups When No Label Was Offered During Training

\begin{tabular}{|c|c|c|c|c|}
\hline Trial & Whole & Patterned part & Difference & $\begin{array}{c}\text { Effect size } \\
(d)\end{array}$ \\
\hline \multicolumn{5}{|c|}{ 12-month-olds $(n=28)$} \\
\hline Salience & $2.65(0.15)$ & $2.39(0.14)$ & 0.26 & 0.4 \\
\hline Test & $2.27(0.09)$ & $1.72(0.08)$ & 0.55 & 0.9 \\
\hline \multicolumn{5}{|c|}{ 19-month-olds $(n=20)$} \\
\hline Salience & $2.88(0.17)$ & $2.48(0.16)$ & 0.40 & 0.7 \\
\hline Test & $2.58(0.10)$ & $2.01(0.08)$ & 0.57 & 1.1 \\
\hline
\end{tabular}

Note. No differences reached significance according to $t$ test against chance responding. Effect sizes were calculated as follows: $d=$ difference/ (average $S D$ ). linguistic input from the experimenter during the training phase that caused children's looking times to differ from baseline preference (as measured in salience trials) and new-label trials. It was the labeling that brought about the infants' responses: Only when a novel word was introduced, trained, and requested did children's looking times to the whole object differ significantly by exceeding their looking times to the part. This suggests that it was something about the label itself that caused infants to associate it with the whole object.

\section{General Discussion}

We conducted three experiments to investigate whether 12- and 19-month-olds, whose expressive vocabularies are still in the beginning stages of development, would construe a novel label as applying to a whole multipart object, rather than its patterned (separable and salient) part, in an ambiguous situation. The selection of a whole object that was arguably two separate objects was a strong test of the breadth of children's tendency to label whole objects. Although prior research (e.g., Saylor et al., 2002) used objects that had undetachable parts, the present experiments asked children to construe two separate objects that fit together in a specific way as a single object. When presented with whole object versus the patterned part (Experiment 1), infants at both ages preferred to associate a novel label with the whole. This preference was also demonstrated when the whole was contrasted with the base part in Experiment 2, ruling out the possibility that the label had been associated with the base in Experiment 1. It appears that it was specifically the use of a label that created this effect. When a label was not presented, in Experiment 3, infants showed no evidence of a preference, suggesting that the bias toward the whole was created by the label training and was, apparently, driven by the process of labeling itself. These results suggest that this tendency is not, at these ages, purely the result of a perceptual preference for contiguous objects.

What could account for infants' tendency to label whole objects as early as 12 months of age? Perhaps this tendency is learned. Recent models of development have hypothesized that such heuristics can be learned generalizations from the process of word learning itself. The emergentist coalition model of Hollich et al. (2000) hypothesizes that infants construct the word learning biases that later become the engines of further word learning through a process called guided distributional learning. The work of Smith (1999; Samuelson \& Smith, 1998) has demonstrated that the tendency to extend a word on the basis of shape and the ability to recognize abstract representations of objects (Smith, 2003) only appears after infants have learned a requisite number of word-toworld mappings. The 12-month-olds in our study did have sizable comprehension vocabularies (from 65 to 110 words, on average). In addition, if we split the data as Smith (2003) did, the 19-montholds with the highest productive vocabulary $(n=16$; productive vocabulary of more than 100 words) appeared to show the strongest preference for the whole object at test (whole minus part $=$ $1.29, S D=1.09$ ) compared with those with the lowest vocabulary ( $n=16$; productive vocabulary of fewer than 26 words; whole minus part $=0.80, S D=0.83$ ), although this difference merely trended toward significance, $t(15)=1.65, p=.12$. Thus, the tendency to label whole complex objects could have been learned. 
Alternative explanations for our findings are also possible. For example, the results of $\mathrm{Xu}$ and Baker (2005) support the idea that infants may be using the labels as a cue to category membership. At 12 months of age, infants use the number of distinct labels to individuate distinct objects, expecting to find the number of objects (up to two) that corresponds to the number of labels they have heard. Thus, the fact that our participants were offered only one label may have signaled to them that we were creating a single object category. Alternatively, those theories that emphasize early sensitivity to the intentions of others (Bloom, 2000; Malle, Moses, $\&$ Baldwin, 2001) might suggest that the youngest infants in our study were using social cues given by the experimenter to ascertain that the experimenter intended to label the whole object. One could ask whether this effect is specific to labels at all. What if a musical motif were played instead of a label? Would musical notes bind these object parts together into a whole object as well? It is likewise possible that if the experimenter had said, during the labeling phase, "My uncle gave this to me" (as in Markson \& Bloom, 1997), perhaps infants would have gone for a whole object interpretation as well. Alternatively, it is possible that infants would also associate the label to the whole object spontaneously, without any training. Perhaps the mere presence of a label at test is the key ingredient that shifts infants' focus to whole objects. Notice, however, that even if this is the case, infants are exhibiting a tendency to label the whole in the absence of any prior salience preference. Thus, whether the effect of a label is happening in the training phase or at test, it is clear that in the absence of a label, infants do not inherently prefer a whole object over a patterned part.

We consider one last, admittedly speculative, explanation. One well-known property of early memory is that it is context sensitive: Children package more information than adults would find necessary in their representations. For example, at 3 months of age, infants have been shown to pay attention to apparently irrelevant contextual information: remembering events such as the color of the crib walls when making the link between kicking their legs and making a mobile move (Rovee-Collier, 1999). Perhaps, in a similar manner, infants make overly broad initial interpretations of new words. When a label is given, infants associate it to all the original stimuli: the whole object, its parts, the movement of the object, the hand holding the object, the person saying the label, and even the color of the walls (the whole complex, à la Vygotsky, 1962). Parallel findings are available from the apparently irrelevant details infants remember during speech segmentation (Houston \& Jusczyk, 2000) and from research on spatial relations by Quinn (2003). Although such context-based sensitivity is not maintained because it fails to allow for the flexible use of information, an early representational bias toward a broad initial interpretation may be an excellent way to begin the learning process. Thus, it is possible that labels act to link disparate components of a scene (e.g., Waxman \& Markow, 1995) and even relations among components together (Casasola, 2005) - more so for children than for adults. In our task, when given a choice, infants prefer the whole over a part, just as they may prefer the whole in its original context over the whole alone. In essence, infants are simply looking for the most consistent correlation between the original scene and the original label (Akhtar \& Montague, 1999; Booth \& Waxman, 2003; Gogate \& Bahrick, 1998; Waxman \& Booth, 2001). This idea suggests some interesting experimental possibilities. For example, if given a choice between the whole and the whole plus context (the background), infants may prefer the whole plus context. Although additional research would be needed to separate such an account from the other explanations, it is intriguing because it considers how an apparent tendency to label wholes may have its origins in a domain-general property of memory rather than an innately specified constraint.

We would be remiss if we did not discuss the difference between associating and interpreting. In our task, infants looked longer at the whole object, indicating that, when given a choice, they more strongly associate the label with the entire complex than any single part. This is not the same as insisting that that label apply only to the whole object. Do infants assume that the part cannot indicate the whole? Although the current experiments cannot speak to this issue, we believe it is doubtful. Certainly, in the apparent absence of a whole, the parts themselves are predictive that the whole may be present. It would be a foolish animal that did not expect the whole when it first caught a glimpse of a tiger's eye. Indeed, Shimpi (2006) found that mothers will often use parts as cues to the absent whole (e.g., "Where's the rest of this toy?"), and Poulin-Dubois and Sissons (2002) found that 18-month-old infants will select a previously labeled object that is missing a prominent part over an intact distractor object. It is thus likely that in the absence of any better referent, infants will also associate the part with the label.

\section{Conclusions}

Regardless of the additional situations in which the tendency to label whole objects may be found, or the final mechanism (or combination of mechanisms) responsible for this tendency, the present experiments represent a stringent test of its breadth across ages and stimuli. They are the first to show that 19-month-olds, and even 12-month-olds, are in possession of a tendency that transcends mere perceptual preference. Infants preferred to map a novel label to a whole object versus either part even though (a) perceptual factors cued the presence of two distinct contiguous parts and (b) one part was perceptually salient. Despite such perceptual cues, infants associated the original label with the whole complex object. This suggests a significantly broader and younger operation of the tendency to label wholes than previously imagined.

\section{References}

Akhtar, N., \& Montague, L. (1999). Early lexical acquisition: The role of cross-situational learning. First Language, 9, 347-358.

Baldwin, D. A. (1989). Priorities in children's expectations about object label reference: Form over color. Child Development, 60, 1289-1306.

Baldwin, D. A. (1991). Infants' contribution to the achievement of joint reference. Child Development, 62, 875-890.

Bloom, P. (2000). How children learn the meanings of words. Cambridge, MA: MIT Press.

Booth, A. E., \& Waxman, S. R. (2003). Mapping words to the world in infancy: Infants' expectations for count nouns and adjectives. Journal of Cognition and Development, 4, 357-381.

Carey, S., \& Xu, F. (2001). Beyond object-files and object tracking: Infant representations of objects. Cognition, 80, 179-213.

Casasola, M. (2005). Can language do the driving? The effect of linguistic input on infants' categorization of support spatial relations. Developmental Psychology, 41, 183-192. 
Clark, E. (1983). Meanings and concepts. In J. H. Flavell \& E. M. Markman (Eds.), Handbook of child psychology, Vol. 3:, Cognitive development (pp. 787-840). New York: Wiley.

Deák, G. O. (2000). Hunting the fox of word learning: Why "constraints" fail to capture it. Developmental Review, 20, 29-80.

Fagan, J., Singer, L., Montic, J., \& Shepard, P. (1986). Selective screening device for the early detection of normal or delayed cognitive development in infants at risk for later mental retardation. Pediatrics, 78, 1021-1026.

Fenson, L., Dale, P., Reznick, S., Bates, E., Thai, D., \& Pethick, S. (1994). Variability in early communicative development. Monographs of the Society for Research in Child Development, 59(Serial No. 242).

Gentner, D. (1978). On relational meaning: The acquisition of verb meaning. Child Development, 49, 988-998.

Gentner, D. (1982). Why nouns are learned before verbs: Linguistic relativity versus natural partitioning. In K. Bean (Ed.), Language, thought, and culture (pp. 301-334). Hillsdale, NJ: Erlbaum.

Gentner, D. (2006). Why verbs are hard to learn. In K. Hirsh-Pasek \& R. M. Golinkoff (Eds.), Action meets word: How children learn verbs (pp. 544-564). New York: Oxford University Press.

Giralt, N., \& Bloom, P. (2000). How special are objects? Children's reasoning about objects, parts, and holes. Psychological Science, 6, 497-501.

Gogate, L. J., \& Bahrick, L. E. (1998). Intersensory redundancy facilitates learning of arbitrary relations between vowel sounds and objects in seven-month-old infants. Journal of Experimental Child Psychology, 69, 133-149.

Golinkoff, R. M., Hirsh-Pasek, K., Cauley, K. M., \& Gordon, L. (1987). The eyes have it: Lexical and syntactic comprehension in a new paradigm. Journal of Child Language, 14, 23-45.

Golinkoff, R. M., Mervis, C., \& Hirsh-Pasek, K. (1994). Early object labels: The case for a developmental lexical principles framework. Journal of Child Language, 21, 125-155.

Hall, D., Waxman, S., \& Hurwitz, W. (1993). How 2- and 4-year-old children interpret adjectives and count nouns. Child Development, 64, 1651-1664.

Hirsh-Pasek, K., \& Golinkoff, R. M. (1996). The origins of grammar: Evidence from early language comprehension. Cambridge, MA: MIT Press.

Hollich, G., Hirsh-Pasek, K., \& Golinkoff, R. M. (1998). Introducing the 3-D intermodal preferential looking paradigm: A new method to answer an age-old question. In C. Rovee-Collier (Ed.), Advances in infancy research (Vol. 12, pp. 355-373). Norwood, NJ: Ablex.

Hollich, G., Hirsh-Pasek, K., \& Golinkoff, R. M. (2000). Breaking the language barrier: An emergentist coalition model for the origins of word learning. Monographs of the Society for Research in Child Development, 65(3, Serial No. 262).

Houston, D. M., \& Jusczyk, P. W. (2000). The role of talker-specific information in word segmentation by infants. Journal of Experimental Psychology: Human Perception and Performance, 26, 1570-1582.

Huttenlocher, J. E., \& Smiley, P. (1987). Early word meanings: The case of object names. Cognitive Psychology, 19, 63-89.

Imai, M., \& Gentner, D. (1997). A crosslinguistic study of early words' meaning: Universal ontology and linguistic influence. Cognition, 62, $169-200$.

Kersten, A. W., \& Smith, L. B. (2002). Attention to novel objects during verb learning. Child Development, 73, 93-109.

Kobayashi, H. (1998). How 2-year-old children learn novel part names of unfamiliar objects. Cognition, 68, B41-B51.

Landau, B., Smith, L. B., \& Jones, S. (1988). The importance of shape in early lexical learning. Cognitive Development, 3, 299-321.

Landau, B., Smith, L. B., \& Jones, S. (1992). Syntactic context and the shape bias in children's and adults' lexical learning. Journal of Memory and Language, 31, 807-825.
Macnamara, J. (1972). Cognitive basis of language learning in infants. Psychological Review, 79, 1-13.

Macnamara, J. (1982). Names for things. Cambridge, MA: MIT Press.

Malle, B. F., Moses, L. J., \& Baldwin, D. A. (Eds.). (2001). Intentions and intentionality. Cambridge, MA: MIT Press.

Markman, E. (1989). Categorization and naming in children: Problems of induction. Cambridge, MA: MIT Press.

Markman, E., \& Hutchinson, J. E. (1984). Children's sensitivity to constraints on word meaning: Taxonomic vs. thematic relations. Cognitive Psychology, 16, 1-27.

Markman, E., \& Wachtel, G. (1988). Children's use of mutual exclusivity to constrain the meaning of words. Cognitive Psychology, 20, 121-157.

Markson, L., \& Bloom, P. (1997, February 27). Evidence against a dedicated system for word learning in children. Nature, 385, 813-815.

Masur, E. V. (1997). Maternal labeling of novel and familiar objects: Implications for children's development of lexical constraints. Journal of Child Language, 24, 427-439.

Merriman, W. E. (1999). Competition, attention, and young children's lexical processing. In B. MacWhinney (Ed.), The emergence of language (pp. 331-358). Mahwah, NJ: Erlbaum.

Mervis, C. B. (1990). Operating principles, input, and early lexical development. Communicazioni Scientifiche di Psicologia Generale, 4, 31-48.

Nelson, K. (1988). Constraints on word learning? Cognitive Development, 3, 221-246.

Poulin-Dubois, D., \& Sissons, M. E. (2002). Is this still called a dog? 18-month-olds' generalization of familiar labels to unusual objects. Infant and Child Development, 11, 57-67.

Pruden, S. M., Hirsh-Pasek, K., Golinkoff, R. M., \& Hennon, E. A. (2006). The birth of words: Ten-month-olds learn words through perceptual salience. Child Development, 77, 266-280.

Quine, W. V. O. (1960). Word and object. Cambridge, England: Cambridge University Press.

Quinn, P. C. (2003). Concepts are not just for objects: Categorization of spatial relation information by infants. In D. H. Rakison \& L. M. Oakes (Eds.), Early category and concept development: Making sense of the blooming, buzzing confusion (pp. 50-76). Oxford, England: Oxford University Press.

Rovee-Collier, C. (1999). The development of infant memory. Current Directions in Psychological Science, 8, 80-85.

Samuelson, L. K., \& Smith, L. B. (1998). Memory and attention make smart word learning: An alternative account of Akhtar, Carpenter, and Tomasello. Child Development, 69, 94-104.

Saylor, M., Sabbagh, M., \& Baldwin, D. (2002). Children use whole-part juxtaposition as a pragmatic cue. Developmental Psychology, 38, 9931003.

Saylor, M., \& Sabbagh, M. A. (2004). Different kinds of information affect word learning in the preschool years: The case of part-term learning. Child Development, 75, 395-408.

Schafer, G., \& Plunkett, K. (1998). Rapid word learning by fifteen-montholds under tightly controlled conditions. Child Development, 69, 309320.

Shimpi, P. (2006). The role of reminders in infants' understanding of absent reference. Unpublished doctoral dissertation, University of Chicago.

Shipley, E. F., \& Shepperson, B. (1990). Countable entities: Developmental changes. Cognition, 34, 109-136.

Smith, L. B. (1999). Children's noun learning: How general learning processes make specialized learning mechanisms. In B. MacWhinney (Ed.), The emergence of language (pp. 277-304). Mahwah, NJ: Erlbaum.

Smith, L. B. (2003). Learning to recognize objects. Psychological Science, 14, 244-250.

Smith, L. B., Jones, S. S., \& Landau, B. (1992). Count nouns, adjectives, 
and perceptual properties in children's novel word interpretations. Developmental Psychology, 28, 273-286.

Soja, N., Carey, S., \& Spelke, E. (1991). Ontological categories guide young children's inductions of word meaning: Object terms and substance terms. Cognition, 38, 179-211.

Spelke, E. (1990). Principles of object perception. Cognitive Science, 14, 29-56.

Vygotsky, L. (1962). Thought and language. Cambridge, MA: MIT Press.

Waxman, S. R., \& Booth, A. E. (2001). Seeing pink elephants: Fourteenmonth-olds' interpretations of novel nouns and adjectives. Cognitive Psychology, 43, 217-242.

Waxman, S. R., \& Kosowski, T. D. (1990). Nouns mark category relations: Toddlers' and preschoolers' word-learning biases. Child Development, $61,1461-1473$
Waxman, S. R., \& Markow, D. B. (1995). Words as invitations to form categories: Evidence from 12-month-old infants. Cognitive Psychology, $29,257-302$.

Woodward, A. (1993). The effect of labeling on children's attention to objects. In E. V. Clark (Ed.), Proceedings of the 24th Annual Child Language Research Forum (pp. 35-47). Stanford, CA: Center for the Study of Language and Information.

Xu, F., \& Baker, A. (2005). Object individuation in 10-month-old infants using a simplified manual search method. Journal of Cognition and Development, 6, 307-323.

Received March 29, 2005

Revision received November 26, 2006

Accepted January 8, 2007

\section{Call for Papers: Special Section on the Interplay of Biology and Environment}

Developmental Psychology invites manuscripts for a special section on the interplay of biology and environment. We are interested in papers that have the potential to change or challenge how developmental psychologists think by gaining new insights into any of the following:

- How experience affects mind, brain, and gene expression throughout development (e.g., how early experience can change gene expression),

- Genetic mediation of environmental effects on mind and body during development (e.g., how similar experiences can have different effects because of the genotypes of those undergoing the experiences),

- How social relations affect cognition, perception, and emotional and physical health (e.g., neuroimaging evidence of the effect of social connectedness or isolation on the brain during development),

- Neuroscientific insights into cognitive, perceptual, emotional, and social processes during development (e.g., evidence that neural systems recruited to do the same chore change over development),

- Interrelations between physical health and mental health (cognitive and emotional) during development (e.g., work in developmental psycho-neuro-immunology), and

- How emotions affect brain function (and hence cognition and perception) and physical health during development (e.g., evidence that one's emotional state affects the way the brain processes stimuli even from earliest infancy).

We would particularly like to encourage submissions from disciplines outside of developmental psychology whose interdisciplinary work holds important implications for understanding developmental processes.

Initial inquiries regarding the special section may be e-mailed to Adele Diamond, Associate Editor, at Adele.Diamond@ubc.ca.

The submission deadline is September 30, 2007. Review papers, empirical reports, and theoretical papers are all encouraged. The main text of empirical reports should not exceed 20 double-spaced pages (approximately 5,000 words), in addition to figures, tables, references, and/or appendixes. Formal submissions must be submitted through the electronic portal of Developmental Psychology at http://www.apa.org/journals/dev/submission.html. Please be sure to specify in the cover letter that your submission is intended for the special section. In the cover letter please also indicate possible reviewers and clearly state how your manuscript will change or challenge the thinking of developmental psychologists. Papers that cannot address the second point will not be accepted for review for the special section, but will be diverted to the regular pool of submissions. 